(2) Open Access Full Text Article

\title{
Ocular pharmacokinetics and tolerability of bimatoprost ophthalmic solutions administered once or twice daily in rabbits, and clinical dosing implications
}

\author{
This article was published in the following Dove Press journal: \\ Clinical Ophthalmology \\ 28 September 2017 \\ Number of times this article has been viewed
}

\author{
Jie Shen' \\ Margot L Goodkin² \\ Warren Tong ${ }^{2}$ \\ Mayssa Attar ${ }^{3}$ \\ 'Clinical Pharmacology, ${ }^{2}$ Clinical \\ Development, ${ }^{3}$ Clinical Pharmacology, \\ Metabolism and Immunology, \\ Allergan plc, Irvine, CA, USA
}

Purpose: Fixed-combination medications can benefit patients requiring multiple agents to lower their intraocular pressure (IOP), but combining agents with complementary mechanisms of action is challenging if their dosing frequency differs. This study compares in vivo pharmacokinetic and ocular tolerability of bimatoprost $0.01 \%$ ophthalmic solutions dosed once or twice daily. Reports of twice-daily dosing in glaucoma patients are also reviewed.

Methods: New Zealand White rabbits were administered bimatoprost $0.01 \%$ monotherapy or fixed-combination bimatoprost $0.01 \%$ /brimonidine $0.1 \%$, once or twice daily in both eyes for 4 days. Ocular tissues were harvested and analyzed by liquid chromatography-tandem mass spectrometry. The pharmacokinetic parameters calculated included maximum observed concentration, time to maximum concentration, and area under the concentration-time curve.

Results: Due to extensive metabolism, bimatoprost concentration was below the quantitation limit by 1 hour post-dose in all samples. Bimatoprost acid exposure, however, could be measured up to 6-8 hours post-dose and was similar in the aqueous humor and iris-ciliary body (pharmacological site of action) of animals treated once or twice daily with either bimatoprost $0.01 \%$ or fixed-combination bimatoprost $0.01 \%$ /brimonidine $0.1 \%$. Increasing dosage frequency in rabbits did not raise the incidence of drug-related conjunctival hyperemia (most common adverse event associated with bimatoprost use in humans), suggesting comparable ocular tolerability of the once- and twice-daily regimens for each formulation.

Conclusion: Bimatoprost $0.01 \%$ administered once or twice daily as monotherapy and in fixed-combination with brimonidine $0.1 \%$ in rabbits show similar pharmacokinetic profiles of bimatoprost acid, especially in the iris-ciliary body. Key findings from previous clinical studies suggest that by varying the concentration of benzalkonium chloride (a preservative with corneal penetration-enhancing properties), formulations of bimatoprost $0.01 \%$ can be administered once or twice daily. These findings support development of bimatoprost $0.01 \%$-based fixed-dose combination therapies administered twice daily for patients who require multiple adjunctive medications to control their IOP.

Keywords: bimatoprost, pharmacokinetics, glaucoma, intraocular pressure, hyperemia

\section{Introduction}

Elevated intraocular pressure (IOP) is a major risk factor for the development and progression of glaucomatous optic neuropathy in patients with open-angle glaucoma (OAG) and ocular hypertension (OHT). There is a strong correlation between higher levels of IOP and increased visual field loss. ${ }^{1}$ Since their introduction in 1996,
Correspondence: Jie Shen

Clinical Pharmacology, Allergan plc,

2525 Dupont Drive, Irvine,

CA 92612 , USA

Tel +I 7I42464989

Email shen_jie@allergan.com
Clinical Ophthalmology 2017:II |76|-1767

| 76 |

Dovepress in

http://dx.doi.org/10.214710PTH 5143428 (c) (1) (-) 2017 Shen et al. This work is published and licensed by Dove Medical Press Limited. The full terms of this license are available at https://www.dovepress.com/terms.php BY NC and incorporate the Creative Commons Attribution - Non Commercial (unported, v3.0) License (http://creativecommons.org/licenses/by-nd/ $/ .0 /$ ). By accessing the work you
hereby accept the Terms. Non-commercial uses of the work are permitted without any further permission from Dove Medical Press Limited, provided the work is properly attributed. For permission hereby accept the Terms. Non-commercial uses of the work are permitted without any further permission from Dove Medical Press Limited, provided the work is properly attributed. For permission
for commercial use of this work, please see paragraphs 4.2 and 5 of our Terms (https://www.dovepress.com/terms.php). 
prostaglandin analog (PGA)/prostamide ophthalmic solutions have become the first-line therapy of choice as they provide safe, effective, 24-hour IOP lowering. ${ }^{2-6}$ If initial monotherapy does not lower the IOP sufficiently, a second drug with a complementary mechanism of action is frequently added to the regimen (eg, a topical $\beta$-blocker added to a PGA or vice versa). If IOP is still not sufficiently lowered, then a third drug may be added. The need for many patients to use more than one hypotensive agent to reach/maintain target IOP has spurred the development of dual and - recently - triple fixed-combination formulations. , $^{27,8}$

Fixed-dose medication combinations have the potential to provide various benefits to patients requiring multiple IOP-lowering medications. ${ }^{9}$ Many patients struggle with maintaining adherence to their medication regimens, due to the sometimes very complex, inconvenient regimens required when using several different monotherapies as adjunctive treatments multiple times per day. ${ }^{10,11}$ By combining pharmacologic agents with complementary mechanisms of action into one bottle, the dosing regimen can be simplified. This may increase convenience of adjunctive treatment, and with that, potentially improve treatment adherence as well. However, fixed combinations containing a PGA/prostamide and a complementary ocular hypotensive agent present a dosing frequency challenge; the PGAs/prostamides are dosed once daily, whereas the adjunctive medications are typically administered two or more times per day. ${ }^{12-14}$ The prostamide bimatoprost is administered as a once-daily drug. ${ }^{15}$ In two identically designed, Phase III pivotal studies supporting the efficacy and safety of bimatoprost $0.03 \%$ ophthalmic solution (preserved with $50 \mathrm{ppm}$ benzalkonium chloride [BAK]), once-daily dosing was found to be optimal for a monotherapy formulation, with twice-daily dosing offering no efficacy advantage. ${ }^{16-20}$ The currently available bimatoprost $0.01 \%$ formulation (containing $200 \mathrm{ppm}$ BAK) has been found to be as effective as the $0.03 \%$ formulation when administered once daily, ${ }^{21-24}$ most likely because higher BAK concentrations were shown to enhance corneal penetration of bimatoprost. ${ }^{25}$

For patients who need to use multiple medications, development of fixed combinations that contain bimatoprost with a medication that requires dosing multiple times per day could be very beneficial. To elucidate the similarities in IOP-lowering efficacy between once- and twice-daily dosing regimens, we present herein the findings from an in vivo comparative pharmacokinetic and ocular tolerability study of bimatoprost $0.01 \%$ dosed once versus twice daily as monotherapy and in a fixed-combination formulation with brimonidine $0.1 \%$, along with clinical correlation from human trials.

\section{Methods}

\section{Animals}

All procedures were performed in compliance with the Animal Welfare Act Regulations (9 CFR 3) and Association for Research in Vision and Ophthalmology (ARVO) Statement for the Use of Animals in Ophthalmic and Vision Research, and approved by the Institutional Animal Care and Use Committee of Covance Laboratories Inc. (Madison, WI, USA). New Zealand White rabbits (Covance Research Products, Denver, PA, USA) were used in the study because they are a common animal model for ocular pharmacokinetic studies and the relative size of their eyes allows excellent visibility of ocular structures. ${ }^{26}$ Healthy female rabbits, 6 months of age, weighing approximately $3 \mathrm{~kg}$ on the first day of dosing were housed in separate cages. Animals were acclimated for 6-14 days prior to dose administration, and maintained in a controlled environment with a 12-hour on/off light cycle; food and fresh water (ad libitum) were provided.

\section{Experimental procedures}

Bimatoprost 0.01\% (200 ppm BAK; pH 7.3; Lumigan ${ }^{\circledR}$ $0.01 \%$ ) ophthalmic solution and fixed-combination bimatoprost $0.01 \% /$ brimonidine $0.1 \%$ (100 ppm BAK; pH 7.0) ophthalmic solution were provided by Allergan plc (Dublin, Ireland). Both formulations were stored at $5^{\circ} \mathrm{C}$ and brought to ambient temperature prior to administration. Gentle inversion ensured homogeneity prior to administration.

Four groups of rabbits (14 per group) were administered either bimatoprost $0.01 \%$ or fixed-combination bimatoprost $0.01 \%$ /brimonidine $0.1 \%$ ophthalmic solutions $(35 \mu \mathrm{L}$ per eye per dose) in both eyes, either once daily or twice daily. In the once-daily groups, a single dose was administered daily for 4 consecutive days. In the twice-daily groups, a single dose was administered twice daily (approximately 12 hours apart) for 3 consecutive days; on Day 4, animals received 1 dose in the morning. Each dose was administered into the eye cul-de-sac via a positive displacement micropipette, ensuring contact with the conjunctiva and cornea. After each dose, the eyelids were gently held together $(5-10 \mathrm{sec})$ to prevent solution loss and distribute the dose across the eye. Each animal was restrained for approximately $1 \mathrm{~min}$ to prevent eye rubbing and was then observed twice daily for any signs of pain and/or distress. Following euthanasia with an intravenous injection of pentobarbital (Sigma-Aldrich Co., St Louis, MO, USA), aqueous humor and iris-ciliary body 
samples were collected from four enucleated eyes in each group at pre-dose (prior to the last dose on Day 4) and hours $0.5,1,2,4,6$, and 8 post-dose on Day 4. Aqueous humor samples were weighed individually following collection. Iris-ciliary bodies were rinsed with saline, blotted dry, and weighed. All samples were kept on dry ice or stored at $-20^{\circ} \mathrm{C}$ until analysis.

\section{Measurement of bimatoprost and bimatoprost acid concentrations}

Iris-ciliary body samples were disrupted for $1 \mathrm{~min}$ using $1 \mathrm{~mL}$ of $1.5 \%$ ammonium hydroxide in methanol:water $(1: 1, \mathrm{v} / \mathrm{v})$ in microtubes filled halfway with $2.3-\mathrm{mm}$ chrome-steel beads (BioSpec Products Inc., Bartlesville $\mathrm{OK}$, USA; or equivalent). After centrifugation, $250-\mu \mathrm{L}$ aliquots of supernatant were mixed with $500 \mu \mathrm{L}$ of internal standard solution (bimatoprost-d4, $50 \mathrm{ng} / \mathrm{mL}$; bimatoprost acid-d4, 100 ng/mL; Cayman Chemical Corp., Ann Arbor, MI, USA) in silanized glass tubes. A $50-\mu \mathrm{L}$ aliquot of $10 \%$ formic acid in water was added, and samples were vortexed for $20 \mathrm{sec}$ before extraction under acidic $\mathrm{pH}$ with $4 \mathrm{~mL}$ of methyl-t-butyl ether. After vortexing, sonicating, vortexing, and centrifugation, the organic phase was removed, dried under nitrogen ( $3 \mathrm{psi} ; \geq 10 \mathrm{~min} ; 40^{\circ} \mathrm{C}$ ), reconstituted in $100 \mu \mathrm{L}$ of acetonitrile, vortexed for $1 \mathrm{~min}$, and a portion was transferred to autosampler vials for injection onto the liquid chromatography-tandem mass spectrometry (LC-MS/ MS) system. Aqueous humor samples were analyzed by direct injection onto the LC-MS/MS system without prior sample processing.

Separation of bimatoprost acid and internal standard was achieved with a Thermo APS-2 Hypersil $3 \mu \mathrm{m}$ column $(50 \times 2.1 \mathrm{~mm})$ using mobile phases A $(0.5 \%$ formic acid in acetonitrile) and $\mathrm{B}(0.5 \%$ formic acid in methanol) according to the following gradient (time in $\mathrm{min} / \% \mathrm{~B}$ ): $0 / 15,1 / 15,2 / 40$, $4.5 / 40,4.51 / 15$, and 6.5/15. Mass spectrometry detection was performed using a PE SCIEX API 5000 mass spectrometer equipped with a turbo ion spray ionization source, with data acquisition in a negative ion multiple reaction monitoring mode. Quantitation was based on the internal standard. The lower limit of quantitation for bimatoprost and bimatoprost acid in the iris-ciliary body was $0.1 \mathrm{ng} / \mathrm{sample}$. The lower limit of quantitation for bimatoprost and bimatoprost acid in the aqueous humor was $0.1 \mathrm{ng} / \mathrm{mL}$.

\section{Ophthalmic examinations}

A board-certified veterinary ophthalmologist conducted an external examination, biomicroscopy, and ophthalmoscopy pre-dose and on study Day 3. Ocular tolerability was assessed daily (approximately 2 hours after the morning dose) by technicians in both eyes, based on irritation scoring using a modified Draize technique. ${ }^{27}$ Two animals per group did not receive any treatment on Day 4 (pre-dose animals) and were evaluated immediately prior to sacrifice. As part of an overall irritation scoring, conjunctival hyperemia/redness was graded 0/normal (vessels normal), 1/mild (vessels definitely injected above normal), 2+/moderate (more diffuse, deeper crimson red, individual vessels not easily discernible), or $3+/$ severe (diffuse bright red).

\section{Pharmacokinetics analysis}

The pharmacokinetic parameters calculated included maximum observed concentration ( $\mathrm{Cmax}$ ), time to maximum concentration (Tmax), and area under the concentrationtime curve (AUC). Analysis was performed using Pharsight Phoenix WinNonlin Version 6.2.1 (St Louis, MO, USA).

\section{Results}

Due to extensive metabolism of bimatoprost in rabbit eyes, bimatoprost concentration in all samples was sporadic and fell below the limit of quantitation by 1 hour post-dose. Bimatoprost acid exposure, however, could be measured up to 8 hours post-dose in aqueous humor samples and up to 6 hours post-dose in iris-ciliary body samples. Exposure was similar in the aqueous humor and iris-ciliary body of animals treated with bimatoprost $0.01 \%$ monotherapy once daily or twice daily (Figure 1A and B; Table 1). In the iris-ciliary body, the concentration of bimatoprost acid was below the limit of quantitation (ie, $<0.1 \mathrm{ng}$ ) at the pre-dose time-point and 6-8 hours post-dose, supporting a lack of dose accumulation with both dosing regimens.

Bimatoprost acid exposure was also similar in the aqueous humor and iris-ciliary body of rabbits treated with dual fixed-combination bimatoprost $0.01 \%$ /brimonidine $0.1 \%$ when administered once daily or twice daily (Figure 1C and D; Table 1). The concentration of bimatoprost acid in the iris-ciliary body was below the limit of quantitation at the pre-dose time-point and by 8 hours post-dose, further supporting a lack of dose accumulation with the once-daily and twice-daily regimens.

Increasing dosage from once to twice daily did not increase the incidence of drug-related conjunctival hyperemia (most common adverse event associated with bimatoprost use in humans) in rabbits (Table 2), suggesting comparable ocular tolerability of the once- and twice-daily regimens for each formulation. In fact, the total incidence of conjunctival hyperemia 

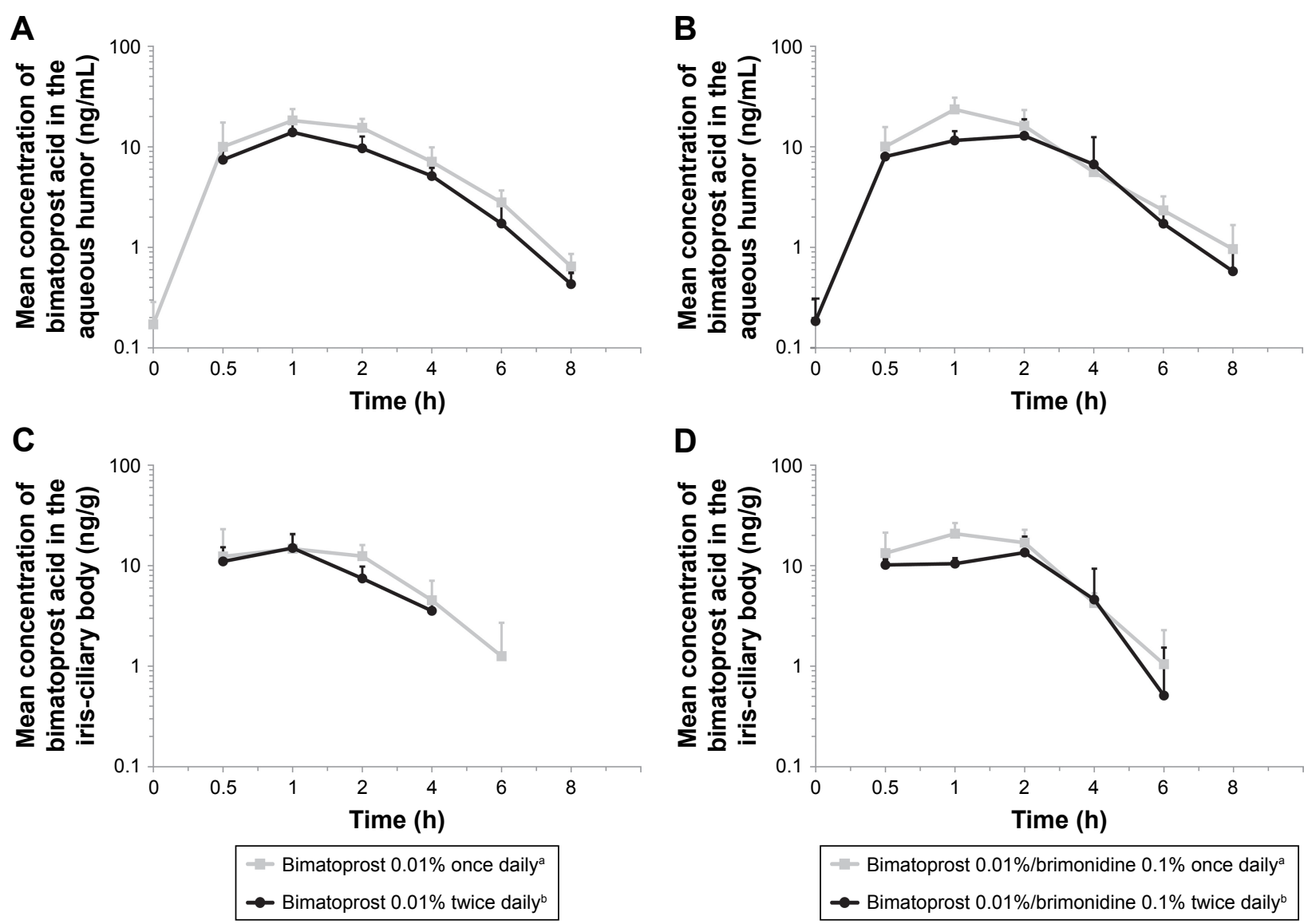

Figure I Mean concentration of bimatoprost acid in the aqueous humor (A, B) and iris-ciliary body (C, D) of New Zealand White rabbits following once- or twicedaily administration of topical bimatoprost $0.01 \%$ or dual fixed-combination bimatoprost $0.01 \% /$ brimonidine $0.1 \%$ ophthalmic solutions. In the aqueous humor and irisciliary body, the lower limits of detection were $0.1 \mathrm{ng} / \mathrm{mL}$ and $1.75 \mathrm{ng} / \mathrm{g}$, respectively. ${ }^{\mathrm{a}}$ Administered once daily for 4 days. ${ }^{\mathrm{b}} \mathrm{Administered}$ twice daily on days I to 3 , and once on day 4 .

appeared to decrease with an increase in dosage, which could be due (at least in part) to the fact that different technicians performed the Draize scoring for the once- and twice-daily groups of each formulation. No drug-related adverse clinical observations were noted over the study duration.

\section{Discussion}

This pharmacokinetic study in rabbits demonstrates that bimatoprost is rapidly absorbed into the eye and hydrolyzed into bimatoprost acid, and that there is a lack of accumulation of bimatoprost acid when bimatoprost ophthalmic solution is administered either once or twice daily. Due to the extensive metabolism of bimatoprost in rabbit eyes, concentrations of bimatoprost were lower than that of bimatoprost acid in aqueous humor and iris-ciliary body.

The comparisons of once- and twice-daily topical administration of bimatoprost $0.01 \%$ or dual fixed-combination bimatoprost $0.01 \% /$ brimonidine $0.1 \%$ in rabbits show

Table I Pharmacokinetic profile of bimatoprost acid in the aqueous humor and iris-ciliary body of New Zealand White rabbits following once- or twice-daily administration of topical bimatoprost $0.01 \%$ or dual fixed-combination bimatoprost $0.01 \% /$ brimonidine $0.1 \%$ ophthalmic solutions

\begin{tabular}{|c|c|c|c|c|c|}
\hline \multirow[t]{2}{*}{ Topical ophthalmic solution } & \multirow{2}{*}{$\begin{array}{l}\text { Dosage } \\
\text { regimen }\end{array}$} & \multicolumn{2}{|l|}{ Aqueous humor } & \multicolumn{2}{|c|}{ Iris-ciliary body } \\
\hline & & $\mathrm{Cmax}(\mathrm{ng} / \mathrm{mL})$ & $\operatorname{AUC}(\mathrm{ng} \cdot \mathrm{h} / \mathrm{mL})^{\mathrm{a}}$ & $\mathrm{Cmax}(\mathrm{ng} / \mathrm{g})$ & AUC (ng.h/g) \\
\hline \multirow[t]{2}{*}{ Bimatoprost $0.01 \%$} & BID & $18.3 \pm 2.7$ & $62.3 \pm 4.8$ & $14.7 \pm 2.7$ & $46.1 \pm 5.1$ \\
\hline & QD & $13.9 \pm 1.8$ & $42.8 \pm 3.1$ & $15.0 \pm 2.8$ & $31.5 \pm 3.0$ \\
\hline Dual fixed-combination bimatoprost & BID & $12.9 \pm 3.0$ & $49.4 \pm 7.4$ & $13.5 \pm 3.0$ & $42.9 \pm 6.6$ \\
\hline $0.01 \% /$ brimonidine $0.1 \%$ & QD & $23.6 \pm 3.6$ & $63.7 \pm 6.3$ & $20.8 \pm 2.9$ & $57.1 \pm 5.4$ \\
\hline
\end{tabular}

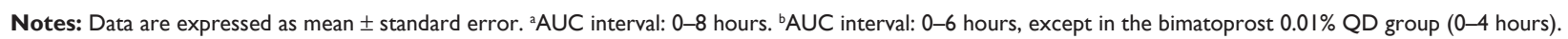
Abbreviations: AUC, area under the concentration-time curve; BID, twice daily; Cmax, maximum observed concentration; QD, once daily. 
Table 2 Incidence of Grade I conjunctival hyperemia in New Zealand White rabbits following BID or QD treatment with topical bimatoprost $0.01 \%$ or fixed-combination bimatoprost $0.01 \% /$ brimonidine $0.1 \%$ a

\begin{tabular}{llllll}
\hline Time-point & \multicolumn{2}{l}{ Bimatoprost 0.0I\% } & & \multicolumn{2}{l}{ Bimatoprost 0.0 I\%/brimonidine 0.1\% } \\
\cline { 2 - 3 } & BID, n eyes (\%) & QD, n eyes (\%) & & BID, n eyes (\%) & QD, n eyes (\%) \\
\hline Pre-dose & $0 / 28(0)$ & $2 / 28(7.1)$ & $0 / 28(0)$ & $1 / 28(3.6)$ \\
Day I & $18 / 28(64.3)$ & $22 / 28(78.6)$ & $5 / 28(17.9)$ & $6 / 28(21.4)$ \\
Day 2 & $1 / 28(3.6)$ & $14 / 28(50.0)$ & $0 / 28(0)$ & $6 / 28(21.4)$ \\
Day 3 & $0 / 28(0)$ & $15 / 28(53.6)$ & $0 / 28(0)$ & $2 / 28(7.1)$ \\
Day 4 & $0 / 16(0)$ & $11 / 16(68.8)$ & $2 / 16(12.5)$ & $1 / 16(6.3)$ \\
Cumulative frequency & $19 / 104(18.3)$ & $64 / 104(61.5)$ & $7 / 104(6.7)$ & $15 / 104(14.4)$ \\
\hline
\end{tabular}

Note: ane report of Grade 2 conjunctival hyperemia in one eye of an animal treated with bimatoprost $0.01 \%$ QD on Day 3.

Abbreviations: BID, twice daily; QD, once daily.

similar pharmacokinetic profiles of bimatoprost acid for both dosing regimens, especially in the iris-ciliary body (site of pharmacological action of bimatoprost). ${ }^{28}$ Although rabbits are known to have a lower blink frequency than humans, ${ }^{29}$ which might result in overestimation of ocular drug exposure following topical eye drop application, the model is well accepted to characterize ocular pharmacokinetics of ophthalmic drugs. ${ }^{26}$ The study design allowed head-to-head comparison of the two dosing regimens, providing meaningful results for extrapolation to humans with faster blinking rates. From a pharmacokinetic/pharmacodynamic standpoint, there was no difference in exposure that would be expected to produce different IOP-lowering effects after once- or twice-daily dosing in a clinical setting. These data thus support the development of twice-daily administration of fixed combinations containing bimatoprost with medications that require a twice-daily dosing frequency.

\section{Clinical correlation with evidence for use of bimatoprost twice daily in humans}

Currently marketed monotherapy formulations of bimatoprost eye drops are optimally administered once per day. Phase III trials of the original monotherapy formulation of bimatoprost $0.03 \%$ in 50 ppm BAK (Lumigan ${ }^{\circledR} 0.03 \%$ ) showed no additional efficacy from being administered twice daily versus once daily; ${ }^{18}$ in fact, once-daily treatment with this formulation was superior to twice-daily treatment. Subsequently, a formulation of bimatoprost $0.01 \%$ in a solution that contains 200 ppm BAK (Lumigan ${ }^{\circledR} 0.01 \%$ ) was developed. This lower concentration of bimatoprost in a higher concentration of BAK was developed in order to increase tolerability to bimatoprost while maintaining the IOP-lowering efficacy of a once-daily formulation. ${ }^{25}$ Preclinical investigations showed that the increase in BAK concentration from 50 to $200 \mathrm{ppm}$ resulted in greater ocular bioavailability for bimatoprost. ${ }^{25}$ At 200 ppm, BAK allows for the lower concentration of bimatoprost $(0.01 \%)$ to be dosed only once daily, while maintaining a therapeutic effect similar to that of bimatoprost $0.03 \%$ in $50 \mathrm{ppm}$ BAK, as demonstrated in the Phase III pivotal registration study of bimatoprost $0.01 \%$ administered once daily. ${ }^{21}$ This suggested that the dosing regimen of the drug could be manipulated depending on the formulation. Additionally, while BAK is known to be associated with ocular side effects such as conjunctival hyperemia, several studies have shown milder and/or fewer reports of conjunctival hyperemia after treatment with bimatoprost $0.01 \%$ in BAK 200 ppm, compared with bimatoprost $0.03 \%$ in BAK 50 ppm. ${ }^{21,24,30,31}$ These findings suggest that a higher BAK concentration does not necessarily lead to a higher rate of conjunctival hyperemia.

Once daily is the preferred administration regimen for a monotherapy formulation; however, the majority of adjunctive medications available are administered more than once per day. Thus, combining bimatoprost with these medications requires adapting the bimatoprost component to use more than once daily. Results of clinical studies performed during development of the once-daily monotherapy formulations of bimatoprost ophthalmic solution suggested that both the concentration of the active ingredient and concentration of the preservative BAK could be modulated with different effects on dosing frequency. ${ }^{25}$ The findings support the concept that bimatoprost $0.01 \%$ ophthalmic solution with $50 \mathrm{ppm}$ BAK may be administered twice daily with similar efficacy as bimatoprost $0.01 \%$ with $200 \mathrm{ppm}$ BAK administered once daily, and without safety or tolerability concerns.

Twice-daily dosing of bimatoprost $0.01 \%$ in humans was studied in two Phase II studies. In the first, preservative-free bimatoprost $0.01 \%$ showed similar IOP lowering when administered once or twice daily in patients with glaucoma or OHT. ${ }^{32}$ Preservative-free bimatoprost $0.01 \%$ was administered once daily for 3 weeks, followed by twice daily for 1 week. Mean IOP change from baseline at $8 \mathrm{AM}$ after 3 weeks of once-daily administration (range, -5.4 to $-5.6 \mathrm{~mm} \mathrm{Hg})^{32}$ was 


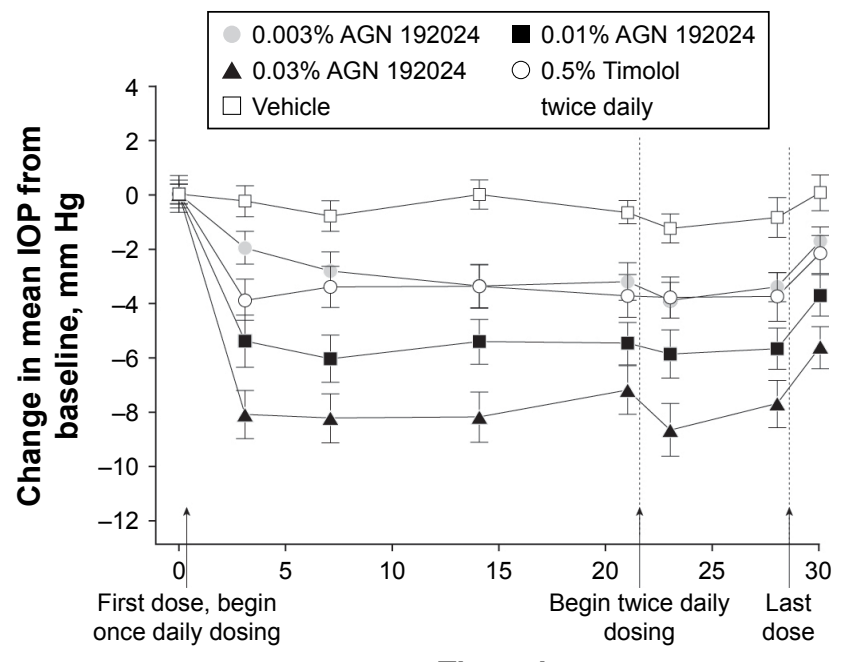

Time, d

Figure 2 Mean (SE) change from baseline intraocular pressure (IOP) at 8 am. A significant decrease $(P \leq .00 \mathrm{I})$ in mean IOP from baseline (day 0 ) was seen in all active treatment groups during the once-daily (days I through $2 I$ ) and twice-daily (days 22 through 28) phases of the study. Baseline mean IOP ranged from 24.5 to $27.0 \mathrm{~mm} \mathrm{Hg}$ among groups $(P=.94)$. The mean decrease from baseline IOP in the $0.03 \%$ AGN 192024 group was significantly greater $(P \leq .02)$ than that in the $0.5 \%$ timolol twice-daily group at every study visit except on day 21 . The mean decrease from baseline IOP in the $0.01 \%$ AGN 192024 group was also significantly greater $(P \leq .04)$ than that with timolol at all study visits except those on days 3 and 30 ( 2 days following the last dose). Reproduced with permission from Arch Ophthalmol. 200 I; I 19(7):994-1000. Copyright (C) (200I) American Medical Association. All rights reserved. ${ }^{32}$ AGN 192024 was developed by Allergan plc.

Abbreviation: SE, standard error.

the same as it was after an additional week of twice-daily administration (range, -5.6 to $-5.8 \mathrm{~mm} \mathrm{Hg} ;{ }^{32}$ Figure 2). Preservative-free bimatoprost $0.01 \%$ twice daily also provided greater than $20 \%$ mean change from baseline in IOP lowering at each time-point (range, $-21.7 \%$ to $-22.5 \%$ change), which again was similar to that of the once-daily administration ( $-20.7 \%$ to $-23.3 \%$ change). ${ }^{32}$ Varying the dosing frequency from once to twice daily also raised no safety or tolerability concerns, ${ }^{32}$ which is supported by other investigational work. ${ }^{33}$ In a separate Phase II study, while there was a numerical advantage to bimatoprost $0.03 \%$ in $50 \mathrm{ppm}$ BAK once daily after 1 month of treatment, there was no statistically significant difference in IOP lowering from baseline versus bimatoprost $0.01 \%$ formulated with 50 ppm BAK, dosed twice daily. ${ }^{25}$

The results of previous studies support the concept that modulating the BAK concentration between formulations drives the once-daily or twice-daily dosing frequency of bimatoprost $0.01 \%$. As glaucoma is a chronic, lifelong disease and many patients eventually require multiple medications to control their IOP, there is a need for fixed-combination ophthalmic solutions containing bimatoprost adjunctive with medications that must be administered more than once daily. Accordingly, a triple fixed-combination of bimatoprost $0.01 \%$, brimonidine $0.15 \%$, and timolol $0.5 \%$ with
50 ppm BAK that can be dosed twice daily is currently in development (Allergan plc), and has now been approved in Mexico. Results showed that the triple fixed-combination provides effective IOP lowering with an acceptable tolerability profile. ${ }^{34,35}$

Based on the nonclinical pharmacokinetic and clinical efficacy data comparisons, our findings support the development of fixed-dose combination therapies containing bimatoprost $0.01 \%$ administered twice daily for patients who require multiple adjunctive medications to control their IOP.

\section{Acknowledgments}

The authors thank Amy Batoosingh and Susan Schneider, $\mathrm{MD}$, of Allergan plc for their contributions to the development of this manuscript. This study was sponsored by Allergan plc (Dublin, Ireland). Writing and editorial assistance was provided to the authors by Michele Jacob, $\mathrm{PhD}$, CMPP, of Evidence Scientific Solutions (Philadelphia, PA, USA) and funded by Allergan plc.

\section{Disclosure}

J Shen, ML Goodkin, W Tong, and M Attar are employees of Allergan plc. The authors report no other conflicts of interest in this work.

\section{References}

1. Leske MC, Heijl A, Hussein M, Bengtsson B, Hyman L, Komaroff E, Early Manifest Glaucoma Trial Group. Factors for glaucoma progression and the effect of treatment: the early manifest glaucoma trial. Arch Ophthalmol. 2003;121(1):48-56.

2. European Glaucoma Society. Terminology and guidelines for glaucoma (4th edition). Available at: http://bjo.bmj.com/content/ bjophthalmol/101/4/1.full.pdf. Accessed September 13, 2017.

3. Aptel F, Cucherat M, Denis P. Efficacy and tolerability of prostaglandin analogs: a meta-analysis of randomized controlled clinical trials. J Glaucoma. 2008;17(8):667-673.

4. van der Valk R, Webers CA, Lumley T, Hendrikse F, Prins MH, Schouten JS. A network meta-analysis combined direct and indirect comparisons between glaucoma drugs to rank effectiveness in lowering intraocular pressure. J Clin Epidemiol. 2009;62(12):1279-1283.

5. Lee AJ, McCluskey P. Clinical utility and differential effects of prostaglandin analogs in the management of raised intraocular pressure and ocular hypertension. Clin Ophthalmol. 2010;4:741-764.

6. Lin L, Zhao YJ, Chew PT, et al. Comparative efficacy and tolerability of topical prostaglandin analogues for primary open-angle glaucoma and ocular hypertension. Ann Pharmacother. 2014;48(12):1585-1593.

7. American Academy of Ophthalmology. Primary open-angle glaucoma. Available at: http://www.aaojournal.org/article/S0161-6420(15)01276-2/ pdf. Accessed May 25, 2017.

8. Kass MA, Heuer DK, Higginbotham EJ, et al. The Ocular Hypertension Treatment Study: a randomized trial determines that topical ocular hypotensive medication delays or prevents the onset of primary openangle glaucoma. Arch Ophthalmol. 2002;120(6):701-713; discussion 829-830.

9. Hóllo G, Topouzis F, Fechtner RD. Fixed-combination intraocular pressure-lowering therapy for glaucoma and ocular hypertension: advantages in clinical practice. Expert Opin Pharmacother. 2014;15(12): $1737-1747$. 
10. Olthoff CM, Schouten JS, van de Borne BW, Webers CA. Noncompliance with ocular hypotensive treatment in patients with glaucoma or ocular hypertension: an evidence-based review. Ophthalmology. 2005;112(6):953-961.

11. Robin AL, Covert D. Does adjunctive glaucoma therapy affect adherence to the initial primary therapy? Ophthalmology. 2005;112(5):863-868.

12. Alcon Laboratories Inc. Timolol GFS (timolol maleate ophthalmic gel forming solution) $0.25 \%$ and $0.5 \%$, Sterile topical ophthalmic drops. [Highlights of prescribing information (6/2007)]. Available at: http:// www.fda.gov/ohrms/dockets/ac/08/briefing/2008-4399b1-58\%20 (Timolo1\%20GFS\%20(timolo1)\%20Labeling).pdf. Accessed May 25, 2017.

13. Pfizer Inc. XALATAN ${ }^{\circledR}$ (latanoprost ophthalmic solution) $0.005 \%$. [Highlights of prescribing information (4/2017)]. Available at: http://labeling.pfizer.com/ShowLabeling.aspx?id=613. Accessed May 25, 2017.

14. Allergan plc. ALPHAGAN ${ }^{\circledR} \mathrm{P}$ (brimonidine tartrate ophthalmic solution) $0.1 \%$ and $0.15 \%$. [Highlights of prescribing information (9/2013)] Available at: http://www.allergan.com/assets/pdf/alphaganp_pi.pdf. Accessed May 25, 2017.

15. Allergan plc. LUMIGAN ${ }^{\circledR}$ (bimatoprost ophthalmic solution) $0.01 \%$ for topical ophthalmic use. [Highlights of prescribing information (10/2016)]. Available at: http://www.allergan.com/assets/pdf/ lumigan_pi.pdf. Accessed May 25, 2017.

16. Sherwood M, Brandt J. Six-month comparison of bimatoprost oncedaily and twice-daily with timolol twice-daily in patients with elevated intraocular pressure. Surv Ophthalmol. 2001;45 Suppl 4:S361-S368.

17. Brandt JD, VanDenburgh AM, Chen K, Whitcup SM. Comparison of once- or twice-daily bimatoprost with twice-daily timolol in patients with elevated IOP: a 3-month clinical trial. Ophthalmology. 2001; 108(6):1023-1031; discussion 1032.

18. Higginbotham EJ, Schuman JS, Goldberg I, et al. One-year, randomized study comparing bimatoprost and timolol in glaucoma and ocular hypertension. Arch Ophthalmol. 2002;120(10):1286-1293.

19. Whitcup SM, Cantor LB, VanDenburgh AM, Chen K. A randomised, double masked, multicentre clinical trial comparing bimatoprost and timolol for the treatment of glaucoma and ocular hypertension. $\mathrm{Br} J$ Ophthalmol. 2003;87(1):57-62.

20. Cohen JS, Gross RL, Cheetham JK, VanDenburgh AM, Bernstein P, Whitcup SM. Two-year double-masked comparison of bimatoprost with timolol in patients with glaucoma or ocular hypertension. Surv Ophthalmol. 2004;49 Suppl 1:S45-S52.

21. Katz LJ, Cohen JS, Batoosingh AL, Felix C, Shu V, Schiffman RM. Twelve-month, randomized, controlled trial of bimatoprost $0.01 \%$, $0.0125 \%$, and $0.03 \%$ in patients with glaucoma or ocular hypertension. Am J Ophthalmol. 2010;149(4):661-671. e1.

22. Nixon DR, Simonyi S, Bhogal M, et al. An observational study of bimatoprost $0.01 \%$ in treatment-naïve patients with primary open angle glaucoma or ocular hypertension: the CLEAR trial. Clin Ophthalmol. 2012;6:2097-2103.
23. Pfennigsdorf S, Ramez O, von Kistowski G, et al. Multicenter, prospective, open-label, observational study of bimatoprost $0.01 \%$ in patients with primary open-angle glaucoma or ocular hypertension. Clin Ophthalmol. 2012;6:739-746.

24. Figus M, Nardi M, Piaggi P, et al. Bimatoprost $0.01 \%$ vs bimatoprost $0.03 \%$ : a 12 -month prospective trial of clinical and in vivo confocal microscopy in glaucoma patients. Eye. 2014;28(4):422-429.

25. European Medicines Agency. Assessment report for Lumigan (January 2010). Available at: http://www.ema.europa.eu/docs/en_GB/document_ library/EPAR_-_Assessment_Report_-_Variation/human/000391/ WC500074096.pdf. Accessed May 25, 2017.

26. Tsonis PA, ed. Animal models in eye research. New York: Elsevier Academic Press; 2011.

27. Farage MA, Maibach HI, Andersen KE, et al. Historical perspective on the use of visual grading scales in evaluating skin irritation and sensitization. Contact Dermatitis. 2011;65(2):65-75.

28. Matsuo T, Cynader MS. Localisation of prostaglandin F2 alpha and E2 binding sites in the human eye. Br J Ophthalmol. 1992;76(4): 210-213.

29. Maurice D. The effect of the low blink rate in rabbits on topical drug penetration. J Ocul Pharmacol Ther. 1995;11(3):297-304.

30. Crichton AC, Nixon DR, Simonyi S, et al. An observational study of bimatoprost $0.01 \%$ in patients on prior intraocular pressure-lowering therapy: the Canadian Lumigan((R)) RC Early Analysis Review (CLEAR) trial. Clin Ophthalmol. 2014;8:1031-1038.

31. Myers JS, Vold S, Zaman F, Williams JM, Hollander DA. Bimatoprost $0.01 \%$ or $0.03 \%$ in patients with glaucoma or ocular hypertension previously treated with latanoprost: two randomized 12-week trials. Clin Ophthalmol. 2014;8:643-652.

32. Laibovitz RA, VanDenburgh AM, Felix C, et al. Comparison of the ocular hypotensive lipid AGN 192024 with timolol: dosing, efficacy, and safety evaluation of a novel compound for glaucoma management. Arch Ophthalmol. 2001;119(7):994-1000.

33. Allergan plc. A Safety and Efficacy Study of Fixed-Combination Bimatoprost and Brimonidine in Chronic Glaucoma or Ocular Hypertension. Available at: https:/clinicaltrials.gov/ct2/show/NCT01863953? term $=$ NCT01863953\&rank=1. ClinicalTrials.gov identifier: NCT01863953. Accessed September 5, 2017.

34. Parra JC, Hartleben CM, Batoosingh A, Bernstein P, Goodkin M. Bimatoprost/Brimonidine/Timolol vs Brimonidine/Timolol Fixed Combinations in Glaucoma: A Randomized, Double-Masked Study, presented at the XXXI Congreso Panamericano de Oftalmología, Aug 4-8, 2015, Bogotá, Colombia.

35. Belfort R Jr, Paula JS, Jordao M, Kim T, Chen MY, Goodkin M. Triple Fixed Combination Bimatoprost/Brimonidine/Timolol Versus Dual Fixed Combination Brimonidine/Timolol Ophthalmic Solutions in Glaucoma: A Multicenter, Randomized, Double-Masked Study, presented at the 60th Congresso Brasileiro de Oftalmologia, Sept 3-6, 2016, Goiânia, Brazil; Available at: https://cbo2016.com.br/cbo2016/ programacao/resumo?trabalho=35. Accessed September 5, 2017.
Clinical Ophthalmology

\section{Publish your work in this journal}

Clinical Ophthalmology is an international, peer-reviewed journal covering all subspecialties within ophthalmology. Key topics include: Optometry; Visual science; Pharmacology and drug therapy in eye diseases; Basic Sciences; Primary and Secondary eye care; Patient Safety and Quality of Care Improvements. This journal is indexed on Submit your manuscript here: http://www.dovepress.com/clinical-ophthalmology-journal

\section{Dovepress}

PubMed Central and CAS, and is the official journal of The Society of Clinical Ophthalmology (SCO). The manuscript management system is completely online and includes a very quick and fair peer-review system, which is all easy to use. Visit http://www.dovepress.com/ testimonials.php to read real quotes from published authors. 